\title{
Cerebral Venous Thrombosis
}

\author{
Brig S Kumaravelu*, Maj A Gupta ${ }^{+}$, Brig KK Singh sм, vsм
}

MJAFI 2008; 64 : 355-360

Key Words : Cerebral veins; Cerebral venous sinuses; Thrombosis; Fibrinolysis

\section{Introduction}

$P_{a}$ uerperal cerebral venous thrombosis (CVT) may account for $15-20 \%$ of stroke in the young. However it is the commonest cause of stroke in young women in India [1-3]. Fifty percent of strokes in Indian women are related to pregnancy and the puerperium and $95.5 \%$ of these are due to CVT $[1,3]$. In western countries the incidence of puerperal CVT ranges from 1 in 166610,000 pregnancies [4-6]. Bansal et al [2], reported a higher incidence of these disorders in North India, with a frequency of 4.5 per 1000 obstetric admissions. In Saudi Arabia the incidence was 7 per 1,00,000 patients with the relative frequency in comparison to arterial strokes being 1:62.5 [7]. The annual incidence of CVT is estimated to be 3 to 4 per million population of which $75 \%$ are women [8]. The reported mortality of 5-30\%, will decline with early detection and better treatment [9-11].

\section{Aetiology}

The causes and associations of CVT are similar to those of venous thrombosis elsewhere in the body. It includes changes in the vessel wall e.g. invasion by malignant cells, or inflammation secondary to infection; changes in the blood flow e.g. polycythemia, dehydration or cardiac failure and changes in the coagulability of blood e.g. thrombocythemia, oral contraceptive use, antithrombin III deficiency; protein C; factor V Leiden mutations, DIC. In pregnancy, CVT is frequently associated with pelvic or deep venous thrombosis due to a hypercoagulable state, combined with the venous stasis, rather than embolism via Bateson's plexus [9].

In the present era, the causes of CVT [12-14] can be grouped broadly as: -

a. Endocrinological disturbances: oral contraceptives, pregnancy, puerperium and androgen therapy/ danazol. b. Hematological/Immunological abnormalities: Antiphospholipid syndrome, anemia, antithrombin deficiency, protein $\mathrm{C}$ deficiency, protein $\mathrm{S}$ deficiency, leukemia, lymphoma, myeloproliferative disorder, paroxysmal nocturnal hemoglobinuria, polycythemia, sickle cell disease and thrombotic thrombocytopenic purpura.

c. Connective tissue and other inflammatory disorders: Behcet's disease, giant cell arteritis, inflammatory bowel disease, Sarcoidosis, systemic lupus erythematosus and Wegener's granulomatosus.

d. Neoplastic: Cerebral metastases, disseminated malignancy, glomus tumour, meningioma and visceral malignancy.

e. Infective: abscess, subdural empyema, meningitis, sinusitis, suppurative otitis media, septicemia, endocarditis, aspergillosis and trichinosis.

f. Miscellaneous: High altitude, cardiac failure, jugular vein ligation, severe dehydration, epoetin alfa therapy, trauma, lumbar puncture, etc.

Infective etiologies (e.g. meningitis, otitis media, subdural haematoma) were over-represented in the early series and accounted for the high morbidity and mortality $(30-50 \%)[9,10,15]$. The mechanism of sinus occlusion is usually either by the development of a prothrombotic state, by direct disturbance of the venous flow (e.g. compression, low flow states) or by direct inflammation of the sinus wall (e.g., the arteritides). Even after full investigation, up to $25 \%$ of patients with venous sinus occlusion fall into "idiopathic" category [15]. In childhood, head and neck disorders especially infections like otitis media and leukemias are the commonest cause $[10,12,16]$.

\section{Pathogenesis}

The postulated mechanisms include thrombosis of cerebral veins which cause local effects due to venous

"Commandant, 155 Base Hospital, 99 APO. ${ }^{+}$Graded Specialist (Medicine), 171 MH, C/o 56 APO. "Dy DGAFMS (P\&T), Min. of Defence, New Delhi

Received : 24.08.06; Accepted : 05.05.08_E mail : neurovelu@yahoo.co.in 


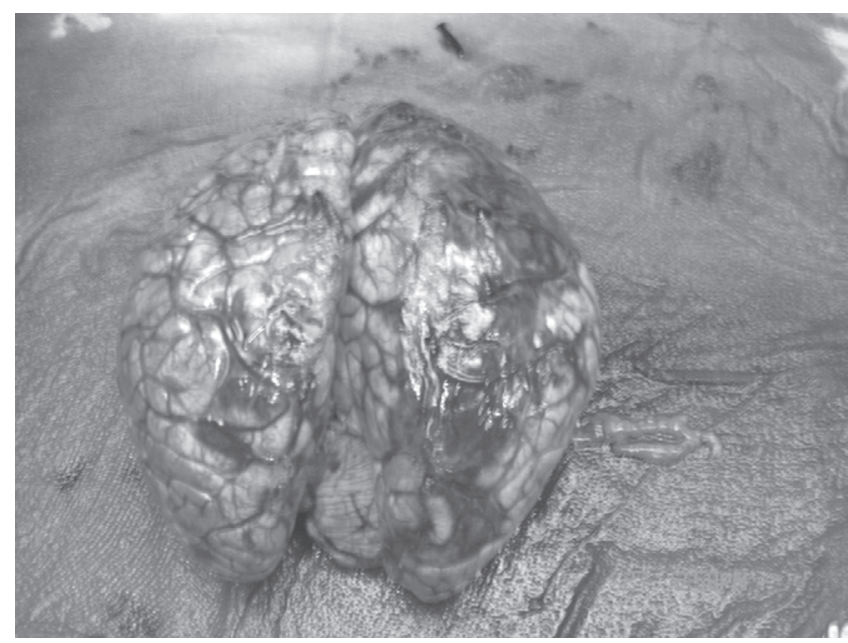

Fig. 1 : Postmortem appearance of one of our patients showing venous hemorrhagic infarcts

obstruction viz. localized cytotoxic cerebral edema and venous infarction [8]. It may start as ischemic neuronal damage, petechiae later merging into large hematomas (Fig.1). Cerebral edema damages the intracellular membrane pumps leading to intracellular swelling and vasogenic edema. CVT results in intracranial hypertension due to obstruction of cerebrospinal drainage in the end, therefore ventricles do not dilate. Intracranial hypertension is seen in $20 \%$ cases of CVT [17].

\section{Clinical Features}

CVT is rare and a high index of suspicion is required to make the diagnosis $[9,10,14,18]$. A good working rule is to consider the possibility of CVT in any condition, which have an association with crural and pelvic vein thrombosis. It can present in protean ways with a variable mode of onset and a wide spectrum of signs $[9,11,18]$. With the growing usage of non-invasive imaging it is apparent that $35 \%$ of patients with benign intracranial hypertension may have underlying CVT [19]. There is a slight female preponderance of 1.3:1 (probably reflecting oral contraceptive usage and pregnancy). Its usual onset is in the $3^{\text {rd }}$ or $4^{\text {th }}$ decades $[1,7]$.

\section{Lateral Sinus Thrombosis}

Thrombosis of the lateral sinus is usually secondary to otitis media and mastoiditis. It is commonly seen in infants and children [14]. Septicemia most commonly occurs with hemolytic streptococci and is present in about $50 \%$ of the patients. Petechiae in the skin and mucous membranes and septic embolism of the lungs, joints, and muscles are infrequent complications of septicemia.

The classic symptoms of lateral sinus thrombosis are fever, headache, nausea and vomiting. These signs are due to increased pressure and are common when the right sinus is occluded, which drains the greater portion of blood from the brain. Local signs of thrombosis of the sinus are usually absent, but occasionally there is swelling over the mastoid region with distension of the superficial veins and tenderness over the jugular vein in the neck. Papilledema develops in over $50 \%$ of the patients and is usually bilateral. Drowsiness and coma are not uncommon symptoms. Jacksonian seizures followed by hemiplegia may reflect extension into the veins draining the hemisphere. Diplopia may result from injury to the $6^{\text {th }}$ cranial nerve by increased intracranial pressure or from involvement of the nerve by extension of the infection to the petrous bone. Gradenigo syndrome due to involvement of the $6^{\text {th }}$ nerve and $5^{\text {th }}$ nerve fibers may be present. There may be signs of damage to the $9^{\text {th }}, 10^{\text {th }}, 11^{\text {th }}$ cranial nerve due to pressure on these nerves by the distended jugular vein, as they traverse the jugular foramen or by extension of the infection into the bone (osteomyelitis) surrounding these structures [20].

\section{Cavernous Sinus Thrombosis}

Cavernous sinus thrombosis usually originates in suppurative processes of the orbit, nasal sinuses or upper half of the face. The infection commonly involves only one sinus at the onset but rapidly spreads through the circular sinus of the opposite side. Nonseptic thrombosis of the cavernous sinus is extremely rare. The sinus may be partially or totally occluded by tumor masses, trauma or arteriovenous aneurysms. The onset of a septic thrombosis is usually sudden and dramatic. The patient appears acutely ill and there is high-grade fever. There is pain in the eyes, and the orbits are painful to pressure. The globes are proptosed by orbital edema and chemosis of the conjunctivae and eyelids is usually present. Diplopia follows involvement of the $3^{\text {rd }}$ cranial nerve. The optic discs are swollen, and there are numerous hemorrhages around the disc when the orbital veins are occluded. The corneas are cloudy and ulcers may develop. The pupils may be dilated or small. Pupillary reactions may be lost with diminished visual acuity [14].

\section{Superior Sagittal Sinus Thrombosis}

The superior sagittal sinus is less commonly the site of infective thrombosis. Infections may reach the superior sagittal sinus from the nasal cavities or as secondary extension from the lateral or cavernous sinuses. The sinus may also be occluded by extension of infection from osteomyelitis or from epidural or subdural infection. The superior sagittal sinus is the most common site of non-septic sinus thrombosis associated with dehydration and marasmus in childhood $[12,13]$. Sagittal sinus thrombosis may also be occluded with oral contraceptive use [21], pregnancy [2,5], hemolytic anemia [14], thrombocytopenia, ulcerative colitis, diabetes mellitus, Behcets syndrome, and other diseases.

Non-septic thrombosis also occurs in adults without 
any obvious cause. The general signs are prostration, fever, headache, and papilledema. In children, local signs include edema of the forehead and anterior part of the scalp and engorgement of the veins in the area of the anterior or posterior fontanellae, with the formation of a caput. Focal neurological signs and symptoms may be entirely absent in nonseptic thrombosis, with increased intracranial pressure as the only presenting sign. In contrast, dramatic signs caused by hemorrhage into the cortical white and gray matter almost always accompany extension of clot into larger cerebral veins. Extension into these veins is common in septic thrombosis and also in a high percentage of the non-septic variety. Seizures (often unilateral), hemiplegia, aphasia, or hemianopia may occur. The diagnosis of non-septic thrombosis should be considered in all infants who show signs of increased intracranial pressure and cerebral symptoms during the course of severe nutritional disturbances and cachexia $[12,14]$.

\section{Thrombosis of Other Dural Sinuses}

Thrombosis of the inferior longitudinal, straight and petrosal sinuses or the vein of Galen rarely occur alone. These sites are usually involved by secondary extension of septic or non-septic thrombosis of the lateral, superior sagittal or cavernous sinuses. Those resulting from involvement of the more important sinuses usually mask any signs or symptoms that may be produced by thrombosis of the inferior longitudinal, straight, or petrosal sinuses. Thrombosis of the great vein of Galen may cause hemorrhages in the central white matter of the hemispheres or in the basal ganglia and lateral ventricles.

Patients of CVT may present may present with a thunderclap headache or the worst headache of their life mimicking subarachnoid hemorrhage [18] or they may come with features suggestive of benign intracranial

Table 1

CVT : Neurological features

$\begin{array}{lc}\text { Neurological signs and symptoms } & \text { Frequency (\%) } \\ \text { Recent headache } & 95 \\ \text { Focal deficits } & 46 \\ \text { Paresis } & 40 \\ \text { Dysphasia } & 22 \\ \text { Visuospatial disorder } & 09 \\ \text { Homonymous hemianopia } & 06 \\ \text { Generalized seizures } & 37 \\ \text { Focal seizures only } & 10 \\ \text { Papilledema } & 41 \\ \text { Impaired consciousness (GCS<14) } & 39 \\ \text { Coma (GCS<5) } & 15 \\ \text { Isolated intracranial hypertension } & 20 \\ \text { Transient neurological symptoms } & 14 \\ \text { Brainstem/cerebellar signs } & 12 \\ \text { Visual impairment } & 10\end{array}$

hypertension. Common neurological signs and symptoms are listed in the Table 1 [11].

\section{Investigations}

The confirmation of CVT no longer requires venous phase intra arterial angiography, computed tomography (CT), magnetic resonance imaging (MRI) and magnetic resonance angiography (MRA) or MR venography (MRV) enable noninvasive diagnosis.

The characteristic CT findings of sagittal sinus thrombosis are of a filling defect within the posterior portion of this sinus following contrast administration. This gives rise to the "Empty Delta Triangle Sign" $[9,10]$ (Fig.2). Since this only occurs when the thrombus extends into the posterior part of the sinus, it is seen in only $30 \%$ of the cases [9]. Other signs include a small ventricular system, reduction of the sulcal pattern (due to swelling), hemorrhagic venous infarcts, intense enhancement of the falx and the tentorium (due to developing venous collaterals), thrombosed cortical veins (the cord sign) and low density changes in the basal ganglia (with straight sinus occlusion) [15]. These finding should be confirmed by MRI and MRA.

Although contrast enhanced CT scanning may contribute to the diagnosis of CVT, a "negative" scan does not exclude the diagnosis. Furthermore CT scans are almost always normal in the absence of established venous infarction [10,21]. An appearance similar to an empty delta sign can be found in patients as a normal variant with a high division of superior sagittal sinus. If there is a clinical suspicion of CVT in the presence of a normal enhanced CT examination, then MRI and/or angiography are indicated.

The MRI appearances of CVT evolve over time. In the acute phase (within five days) there is absence of the normal venous flow void on T1 and T2 weighted images and the thrombus itself appears isointense and hypo intense on these sequences, respectively. Within

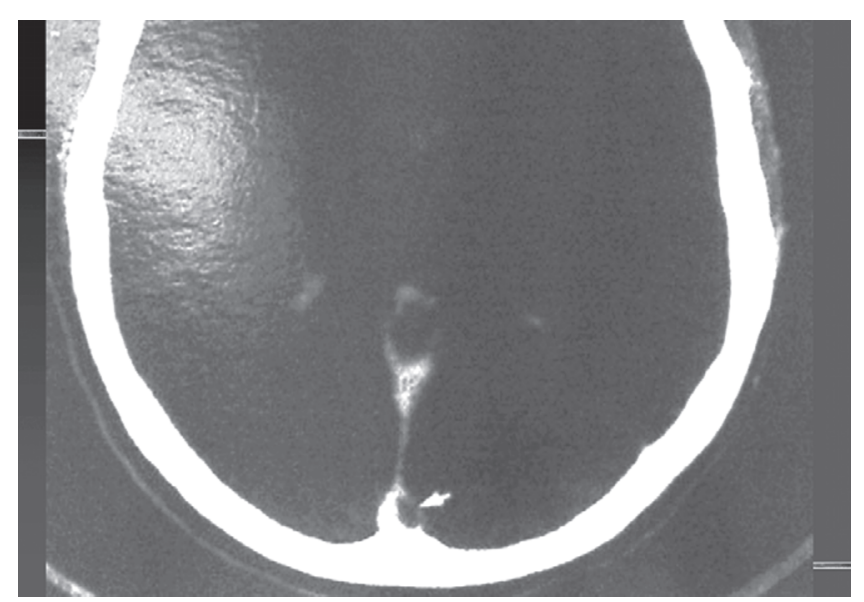

Fig. 2: Contrast enhanced CT scan of head showing the empty Delta sign 


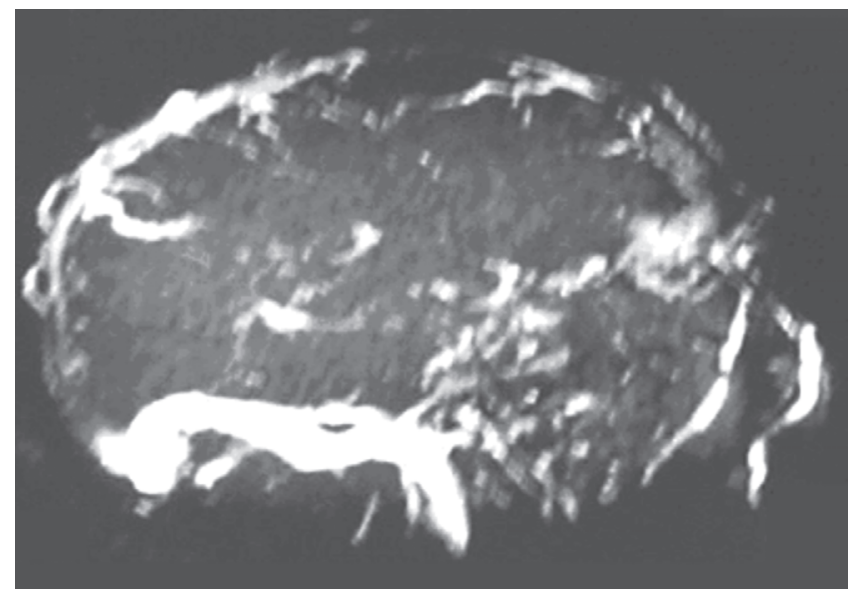

Fig. 3: MR angiography of a patient showing thrombosis of the superior sagittal sinus

the next 10 days, hyperintense signals appear within the occluded sinuses on the $\mathrm{T} 1$ and then the $\mathrm{T} 2$ sequences [10,22]. In continuing obstruction, the signal intensity decreases, whilst in cases of recanalisation the venous flow void reappears. Venous phase MRA shows characteristic filling defects in the principal dural sinuses and does not require the administration of contrast [22](Fig. 3).

Intravenous subtraction angiography requires the injection of a large volume of contrast and provides poor image definition. It is associated with a large incidence (up to 10\%) of systemic reaction due to contrast and only delineates the principal sinuses[10]. Intra-arterial angiography (with or without digital subtraction) provides high definition image but is associated with a small risk (5\%) of neurological complications.

Lumbar puncture may be required to exclude other pathologies such as meningitis or subarachnoid hemorrhage [15]. The CSF in CVT may be normal or there may be increase in proteins, WBCs and red cells. The CSF pressure is significantly raised in these cases (at least $30 \mathrm{~cm}$ of CSF). Finally, repeated lumbar puncture to drain CSF is used rarely as a therapeutic maneuver when vision is threatened $[10,15]$.

The diagnosis of CVT is not enough as it is important to look for the underlying condition, which led to CVT. An attempt should be made to look for a procoagulant state such as Factor V Leiden, Protein C or S deficiency, antithrombin III, antiphospholipid antibody(APLA), hyperhomocystinemia, infections, endocrinopathy, vasculitis or hematological disorder and malignancies. The incidence of procoagulant factors is variable. In unselected patients of venous thrombosis, the incidence of Protein $\mathrm{C}$ deficiency was $10 \%$ in young patients, factor V Leiden mutation in $20-50 \%$ and APLA in $24 \%$ of young strokes [23]. 20-35\% of patients with CVT may be uncharacterized. In a study of 40 patients with
CVT, six cases of thrombophilia (15\%) were found, one each of protein $\mathrm{C}$ and $\mathrm{S}$ deficiency and four of factor $\mathrm{V}$ Leiden mutation. Three (8\%) had anticardiolipin antibodies, one had SLE and two had primary APLA [24]. Many patients had multiple prothrombotic state.

\section{Management}

Treatment of dural sinus thrombosis is influenced by its etiology and the clinical features. In patients with a pure benign intracranial hypertension-like syndrome, diuretics may be sufficient to control the raised intracranial pressure. If these measures fail and visual fields/acuity deteriorate, optic nerve sheath fenestration or lumbo-peritoneal shunt may be required [9].

Anecdotal reports say that anticoagulation with intravenous heparin may be of benefit $[9,11]$. There is preliminary evidence to show that heparin improves outcome and can be administered in the presence of hemorrhagic venous infarction [8,9]. The dramatic response to heparinisation is due to limitation of further thrombogenesis within the superficial cortical veins. In a recent Cochrane review, the authors concluded that anticoagulant treatment for cerebral sinus thrombosis appeared to be safe and was associated with a potentially important reduction in the risk of death or dependency which did not reach statistical significance [25]. The multicentric Dutch-European Cerebral Sinus Thrombosis Trial (DECST) evaluated anticoagulation and benefit with low molecular weight heparin (LMWH) and warfarin and recommended initial therapy with LMWH for 5-7 day, followed by at least three months of warfarin, or more, in cases with an underlying prothrombotic state.

Though fibrinolysis or local administration of thrombolytic agents via catheter should be beneficial in patients with CVT, there is insufficient evidence to support its widespread use. An earlier study group has recommended that in view of its invasive and potentially dangerous nature their use should be restricted to cases with a poor prognosis [11]. As per the recent Cochrane review, there is currently no available evidence from randomised controlled trials regarding the efficacy or safety of thrombolytic therapy in dural sinus thrombosis [26].

In a landmark study on the causes and predictors of mortality the authors reached two conclusions [27]. First, although CVT has a low case fatality, it is possible to predict some patients who are at increased risk of death. These patients should be closely monitored, and worsening of their clinical condition should be regarded as an indication for more aggressive treatment. Second, given the potential for neurologic recovery after CVT, there is a case for assessing decompressive craniectomy 
in patients who are deteriorating due to a parenchymal lesion producing a mass effect.

Earlier series of CVT reported mortalities of $30-50 \%$ [1,9] and left untreated, the condition is potentially life threatening. Recent reports $[11,16,18,27]$ describe mortality of 5-30\%. The most common cause of death was transtentorial herniation due to a unilateral hemorrhagic lesion or diffuses edema and bilateral lesions. Main predictors of death within 30 days were seizure, mental status disturbances, coma $(\mathrm{GCS}<9)$, deep CVT, right sided hemorrhage and posterior fossa lesions [27]. A few of the survivors may develop permanent deficits like focal limb weakness, epilepsy or optic atrophy. Mortality and morbidity associated with CVT should fall further if optimum treatment (antithrombotic, anticoagulants and fibrinolytic) is standardized. The factors associated with a poor outcome are papilledema, impaired consciousness, coma, age more than 33 years, diagnostic delays of 10 days or more, intracerebral hemorrhage and involvement of the straight sinus, while isolated intracranial hypertension and delta sign on CT scan have a better outcome [11].

Recent literature has three multicentric studies on pediatric population [28-30]. A total of 129 children were studied in the USA, Europe and Israel. Age ranged from one day to 13 years, one third being neonates. There was a male predominance. Clinically they had lethargy, anorexia, headache, vomiting, seizures, focal signs and coma with additional fever and respiratory distress in the neonates. Majority had positive prothrombotic work up, protein $\mathrm{C}$ and antithrombin III deficiency and methyl tetrahydrofolate reductase mutation being the commonest. Anticoagulation and antibiotics were given. Mortality was seen in $10 \%$ and sequelae in $60 \%$ cases. Older age, lack of infarction, anticoagulation and lateral and/or sigmoid sinus involvement was associated with good prognosis while coma at presentation was associated with poor prognosis.

\section{Our Experience}

Sixty consecutive patients of CVT were managed over a five-year period (2000-2005) in two referral hospitals of the Armed Forces. All patients were evaluated with relevant investigations including neuroimaging (CT and/or MRI with/without MR venography). Procoagulant workup was undertaken after six months without anticoagulants for at least 14 days. Anticoagulants were given in 54 patients who had no contraindications. The age of patients ranged from 5 to 60 years with a mean of $34.97 \pm 8.64$ years. Majority $(75 \%)$ of our patients were in $3^{\text {rd }}$ and $4^{\text {th }}$ decades of life. Male female ratio was 11:9. Presenting symptoms were headache in 45 patients, focal neurologic deficits
Table 2

Clinical features of CVT

\begin{tabular}{|c|c|c|c|}
\hline $\begin{array}{l}\text { Neurological signs } \\
\text { and symptoms }\end{array}$ & CVST study group & $\begin{array}{l}\text { Frequency (\%) } \\
\text { Saudi study[7] }\end{array}$ & Our study \\
\hline Recent headache & 95 & 82 & 75 \\
\hline Focal deficits & 46 & 27 & 85 \\
\hline Paresis & 40 & - & 85 \\
\hline Dysphasia & 22 & - & - \\
\hline Visuospatial disorder & 9 & 2 & - \\
\hline Homonymous & 6 & - & 4.1 \\
\hline Seizures & 47 & 10 & 36.7 \\
\hline Generalized Seizures & 37 & - & 24 \\
\hline Focal seizures only & 10 & - & 12.7 \\
\hline Papilledema & 41 & 80 & 66.7 \\
\hline $\begin{array}{l}\text { Impaired sensorium } \\
(\mathrm{GCS}<14)\end{array}$ & 39 & 10 & 21 \\
\hline Coma $(\mathrm{GCS}<5)$ & 15 & - & 10 \\
\hline Isolated intracranial & 20 & - & 3.33 \\
\hline TIAs & 14 & - & 1.7 \\
\hline Brainstem/cerebellar & 12 & - & - \\
\hline Meningeal signs & - & 2 & 20 \\
\hline Visual impairment & 10 & - & 8.3 \\
\hline
\end{tabular}

in 38 , seizures in 22 , fever in 4 , jaundice and injury in 2 each. Examination revealed focal neurologic deficits in 51, papilleoedema in 40, meningeal signs in 12 and concomitant deep vein thrombosis in 10. Eight patients did not have any neurologic deficit. They are compared with earlier studies as shown in Table 2.

Evaluation of etiological factors revealed puerperium in 16, anemia in 11, meningitis, oral contraceptive use and systemic lupus erythematosus in three each, antiphospholipid syndrome, protein $\mathrm{C}$ deficiency and protein $\mathrm{S}$ deficiency in two each with one patient showing factor $\mathrm{V}$ Leiden mutation. Two patients came from high altitude without any other risk factors.

CT scan was normal in 19 patients. Venous hemorrhagic infarcts was seen in 20, nonhemorrhagic infarcts in eight, empty delta sign in eight and diffuse cerebral edema in six cases. Magnetic resonance imaging showed thrombosis of superior sagittal sinus in 44 , transverse sinus in 20 , straight sinus in 13 , sigmoid sinus in nine and cavernous sinus in two patients. Isolated venous infarcts were seen in four patients. Twenty-three patients had hemorrhagic infarcts while nine had nonhemorrhagic infarcts. One patient underwent a four vessel digital substraction angiography that showed superior sagittal sinus thrombosis.

Fifty four patients received anticoagulants. Patients with procoagulant factors were given anticoagulants for a longer duration, at times for the rest of their lives. Forty patients recovered fully and 12 had minimal deficit. Five patients died and three had severe neurologic sequelae. 


\section{Conflicts of Interest}

None identified

\section{References}

1. Srinvasan K. Cerebral venous thrombosis. In : JS Chopra, IMS Sawhney, eds. Neurology in Tropics. $1^{\text {st }}$ Ed New Delhi: BI Churchill Livingstone Pvt Ltd, 1999; 497-505.

2. Bansal BC, Gupta RR, Prakash C. Stroke during pregnancy and puerperium in young females below the age of 40 years as a result of cerebral venous/venous sinus thrombosis. Jpn Heart J 1980; 21: 171-83.

3. Srinivasan K. Cerebral venous and arterial thrombosis in pregnancy and puerparium. Angiology 1983; 134: 731-46.

4. Lamy C, Hamon JB,Coste J, Mas JL. Ischemic stroke in young women: Risk of recurrence during subsequent pregnancies. Neurology 2000; 55; 269-74.

5. Kittner SJ, Stern BJ, Feeser BR, Hebel JR, Nagey DA, Buchholz DW, et al. Pregnancy and the Risk of Stroke. N Engl J Med 1996; 335: 768-74.

6. Lanska DJ, Kryscio RJ. Risk factors for puerparium, postpartum stroke and intracranial venous thrombosis. Stroke 2000; 31: 1274-82.

7. Daif, et al. Cerebral venous thrombosis in adults. A study of 40 cases from Saudi Arabia. Stroke 1995; 26: 1193-5.

8. Stam J. Thrombosis of the Cerebral Veins and Sinuses. N Engl J Med 2005; 352: 1791-8.

9. Bousser MG. Cerebral venous thrombosis: diagnosis and management. J Neurol 2000; 247: 252-8.

10. Allogren H, Abbot RJ. Cerebral venous thrombosis. Postgrad Med J 2000; 76: 5-12.

11. De Bruijn SF, de Haan RJ, Stam J. Clinical features and prognostic factors of CVT in a prospective series of 59 patients. J Neurol Neurosurg Psychiatry 2001; 70: 105-8.

12. Huisman TA, Holzman D, Martin E, Willi UV. Cerebral venous thrombosis in childhood. Eur Radiol 2001; 11: 1760-5.

13. De Veber G, Andrew M. Cerebral sinovenous thrombosis in children. N Engl J Med 2001; 345: 417-23.

14. Fishman RA. Cerebral veins and sinuses. In: Rowland LP ed. Merrit's Neurology, $10^{\text {th }}$ ed. Lippincott Williams \& Williams, Philadelphia 2000: 268-71.

15. Martin PJ, Enevoldson TP. Cerebral venous thrombosis. Postgrad Med J 1996; 72: 72-6.

16. Carvalho KS, Bodensteiner JB, Connolly PJ, Garg BP. Cerebral venous Thrombosis in children. J Child Neurol 2001; 16: 57480 .
17. Fero JM, Canhao P, Stam J, Bousser MG, Barinagarrementeria F. Prognosis of cerebral vein and dural sinus thrombosis: results of the International Study on Cerebral Vein and Dural Sinus Thrombosis (ISCVT). Stroke 2004; 35: 664-70.

18. de Bruijn SFTM, Stam J, Kappelle LJ. Thunderclap headache as first symptom of cerebral venous sinus thrombosis. Lancet 1996; 348: 1623-5.

19. Jacobs K, Moulin T, Bogousslavsky J, Woimant F, Dehaene I, Tatu L, et al. The stroke syndrome of cortical vein thrombosis. Neurology 1996; 47: 376-82.

20. Arboix A, Bechich S, Oliveres M, Garcia-Eroles L, Massons J, Targa C. Ischemic stroke of unusual cause: clinical features, etiology and outcome. Eur J Neurol 2001; 8: 133-9.

21. Kemmeren JM, Tanis BC. Risk of arterial thrombosis in relation to oral contraceptives (RATIO) study. Oral contraceptives and the risk of ischemic stroke. Stroke 2002; 33: 1202-8.

22. Vogt TJ, Bergman C, Villringer A, Einhaupt K, Lissner J, Felix $R$. Dural sinus thrombosis: value of venous MR angiography for diagnosis and follow up. AJNR 1998; 62: 1191-8.

23. Bick RL. Heriditary and acquired thrombophilic disorders. Clin Appl Thromb Hemost 2006; 12: 125-35 .

24. Deschiens MA, Conard J, Horellou MH, Ameri A, Preter M, Chedru F, et al. Coagulation studies, Factor V Leiden and Anticardiolipin Antibodies in 40 cases of Cerebral Venous Thrombosis. Stroke 1996; 27: 1724-30.

25. Stam J, De Bruijn SF, De Veber G. Anticoagulation for cerebral sinus thrombosis. Cochrane Database Syst Rev 2002;(4):CD00 2005.

26. Ciccone A, Canhão P, Falcão F, Ferro JM, Sterzi R. Thrombolysis for cerebral vein and dural sinus thrombosis. Cochrane Database Syst Rev 2004; (1):CD00 3693.

27. Canhao P, Ferro JM, Lindgren AG, Bousser MG, Stam J, Barinagarrementeria F; ISCVT Investigators. Causes and predictors of death in cerebral venous thrombosis. Stroke 2005; 36: $1720-5$.

28. Carvalho KS, Bodensteiner JB, Connolly PJ, Garg BP. Cerebral venous thrombosis in children. J Child Neurol. 2001;16:57480 .

29. Kenet G, Waldman D, Lubetsky A, Kornbrut N, Khalil A, Koren A, et al. Paediatric cerebral sinus vein thrombosis. A multi-center, case-controlled study. Thromb Haemost 2004;92:713-8.

30. Sébire G, Tabarki B, Saunders DE, Leroy I, Liesner R, SaintMartin C, et al. Cerebral venous sinus thrombosis in children: risk factors, presentation, diagnosis and outcome. Brain 2005;128:477-89.

\section{NOTICE}

MJAFI is despatched to all officers of AMC and AD Corps (except the non technical officers) as per the addresses and nominal roll forwarded by the units. Any officer not receiving the journal may please forward their name and address as per format given below for inclusion in the mailing list :-

$\begin{array}{lllll}\text { Personal No Rank } & \begin{array}{l}\text { Name in full } \\ \text { (as per Army list) }\end{array} & \begin{array}{l}\text { Appointment } \\ \text { (speciality) }\end{array} & \text { Previous Unit } & \begin{array}{l}\text { Present Address } \\ \text { (with PIN) }\end{array}\end{array}$

